\title{
9th International Symposium on Resistance Arteries (ISRA)
}

\author{
February 17-21, 2008, Hamilton Island, Australia
}

\author{
Shaun L. Sandow ${ }^{\mathrm{a}}$ Christopher R. Triggle ${ }^{\mathrm{b}}$ Michael A. Hill ${ }^{\mathrm{c}}$ \\ a Department of Pharmacology, University of New South Wales, Sydney, N.S.W., Australia; \\ ${ }^{\mathrm{b}}$ Medical Education, Weill Cornell Medical College in Qatar, Education City, Doha, Qatar; \\ 'Dalton Cardiovascular Research Center and Department of Medical Pharmacology and \\ Physiology, University of Missouri, Columbia, Mo., USA
}

The 9th International Symposium on Resistance Arteries (ISRA) was held between the 17th and 21st February, 2008 on Hamilton Island, Australia. Hamilton Island is a Great Barrier Reef Island situated within the Whitsunday Islands off the north east coast of Australia. The meeting, as had been the case for previous ISRAs, brought together a truly international group of scientists with either a direct focus on the resistance vasculature or with areas of interest applicable to resistance artery research. Ten thematic symposia covered areas including cellular communication, ion channels and the regulation of resistance vessel function, mechanotransduction, rhythmicity, vascular dysfunction (diabetes, oxidative stress, vascular remodeling) and the influence of the fetal environment. Aspects of the meeting are briefly described below, while a full listing of abstracts are available through the Journal of Vascular Research (www.karger.com/doi/10.1159/000113929). The complete meeting report is available at www.karger.com/doi/10.1159/000243970.

\section{KARGER}

(C) 2009 S. Karger AG, Basel
Michael A. Hill, $\mathrm{PhD}$

Dalton Cardiovascular Research Center

University of Missouri

134 Research Park Drive, Columbia, MO 65211 (USA)

Tel. +1 573884 4601, Fax +1 573884 4232, E-Mail Hillmi@missouri.edu 
Fundamental to the behavior of resistance arteries is the communication that occurs between endothelial cells and the underlying vascular smooth muscle (VSM). This interaction occurs through direct physical contact such as provided by myoendothelial gap junctions (MEGJs) or via chemical mediators. In relation to MEGJs an important question relates to whether these structures provide low resistance pathways for electrotonic spread of signals or whether they also provide a pathway for transfer of chemical mediators. Beny [1] reviewed passive and regenerated elements of propagated signals with an emphasis on the roles of chemically and electrically coupled pathways. Beny further emphasized the likelihood that VSM membrane potential (Em) likely influences the underlying endothelial cell layer. In contrast, endothelial cell Em would only impact on VSM in arterioles and decrease in importance as vessel diameter increases with a greater number of smooth muscle layers. De Wit [2] described the roles of various connexins $(\mathrm{Cx} 37, \mathrm{Cx} 40, \mathrm{Cx} 43$ and $\mathrm{Cx} 45)$ in communication along the vessel 
wall. Using genetically manipulated mouse models differing connexins were shown to provide distinct pathways for conduction. For example, the $\mathrm{Cx} 40$ deficient mouse exhibits impaired conducted responses to acetylcholine and bradykinin while the conducted response to adenosine was not altered by the lack of the particular connexin. Further specificity was shown by $\mathrm{Cx} 45$ knock-in on the Cx40 knock out background not being able to rescue the impaired ACh conduction response [3].

Ledoux [4] introduced a novel spatially-restricted $\mathrm{Ca}^{2+}$ signaling event termed the ${ }^{\prime} \mathrm{Ca}^{2+}$ pulsar'. Important for these studies was the availability of the endothelial-specific GCaMP2 $\mathrm{Ca}^{2+}$ indicator expressing mouse [5]. The kinetics and pharmacology (inhibited by the non-specific blocker xestospongin $\mathrm{C}$, but not ryanodine) of these events showed them to be separate to other localized $\mathrm{Ca}^{2+}$ release phenomena such as sparks. These $\mathrm{IP}_{3}$-mediated localized events appear important in modulating endothelial function particularly as relates to $\mathrm{Ca}^{2+}$-activated $\mathrm{K}^{+}$channels localized in the vicinity of myoendothelial junctions [6], which corresponds to the spatial localization of $\mathrm{IP}_{3}$ receptors at such sites [7]. In a related presentation, Isakson described an in vitro vascular co-culture system using murine microvascular endothelial and VSM to image $\mathrm{Ca}^{2+}$ dynamics at the myoendothelial junction [8].

A particular focus of the meeting related to signaling mechanisms underlying the myogenic response of small arteries. Although vasoconstriction to the mechanical forces exerted by intraluminal pressure has been recognized for over 100 years (Bayliss, 1901) the membrane events involved in sensing a change in pressure have largely remained obscure. Brayden [9] presented evidence for the involvement of non selective cation channels involving TRP proteins (TRPC3 and TRPC6) as upstream mediators of pressure-induced membrane depolarization [10], which is believed to precede opening of voltage-gated $\mathrm{Ca}^{2+}$ channels and an increase in global cytosolic $\mathrm{Ca}^{2+}$. In addition to being candidate mechanosensors Brayden highlighted other 
mechanisms by which TRP proteins modulate myogenic tone. In cerebral VSM, TRPV4 channels, for example, allow entry of extracellular $\mathrm{Ca}^{2+}$ which stimulates $\mathrm{Ca}^{2+}$ sparks and STOCs (resulting from increased $\mathrm{BK}_{\mathrm{Ca}}$ activity) leading to hyperpolarization and inhibition of myogenic tone. Adding to the significance of these channels in vivo suppression of TRPM4 with antisense oligonucleotides impairs both cerebral vessel myogenic responsiveness and autoregulation [11]. Scholfield et al [12] similarly demonstrated that $\mathrm{Ca}^{2+}$ sparks in retinal arteriolar smooth muscle underlie activation of $\mathrm{BK}_{\mathrm{Ca}}$ channels. This may, however, not occur in all vascular beds as Jackson [13] suggested that in VSM from skeletal muscle sparks are absent and $\mathrm{BK}_{\mathrm{Ca}}$ is not regulated by ryanodine-sensitive $\mathrm{Ca}^{2+}$ release. Supporting this, it was suggested that differences may exist in the subunit composition of $\mathrm{BK}_{\mathrm{Ca}}$ channels across vascular beds [14].

In related studies the role of integrins in arteriolar myogenic reactivity [15] were considered. Using atomic force microscopy Hill et al [10] described studies showing that myogenic-like activity could be elicited at the single smooth muscle level by application of force through a specific matrix protein-integrin interaction. Specific emphasis was placed on the role of fibronectin in arteriolar smooth muscle mechanotransduction and the concept that distinct mechanoreceptor complexes may be formed by different matrix protein - integrin interactions [16].

Stefan Bolz [17] provided further details of the possible involvement of a novel pathway in the myogenic response, namely that involving sphingosine-1-phosphate (S1P). An increase in transmural pressure leads to translocation of sphingosine kinase to the plasma membrane, phopshorylation of sphingosine and generation of the mediator S1P. S1P then participates in the activation of $\mathrm{Ca}^{2+}$ dependent $\left(\mathrm{Ca}^{2+}\right.$-calmodulin) and $\mathrm{Ca}^{2+}$ independent (Rho kinase modulation of myosin phosphatase). Adding to this picture Bolz described the phosphatase involved in inactivating S1P, namely S1P phosphohydrolase. Interestingly, access to extracellular S1P by 
S1PP was suggested to be dependent on the transport function of the cystic fibrosis transporter (CFTR). By transporting S1P across the membrane CFTR decreases availability of the phosphorylated messenger to its receptor and targets it towards the phosphatase for inactivation. Collectively these data suggest an intriguing interaction between SPP1 and CFTR in the regulation of arteriolar tone [18].

A number of presentations centered on the role of various ion channels in resistance vessel function. Aalkjaer's group presented an interesting paper describing a siRNA approach for the in vivo down regulation of the L-type $\mathrm{Ca}^{2+}$ channel in small mesenteric arteries of the rat [19]. This transient tranfection procedure showed a down regulation of mRNA for Cav1.2 by $93 \pm 2 \%$ after three days and was paralleled by decreased protein expression as shown by immunohistochemistry. Jaggar [20] challenged the conventional view that $\mathrm{IP}_{3}$-mediated vasoconstriction occurs solely through $\mathrm{SR} \mathrm{Ca}^{2+}$ release. Specifically, in cerebral artery vascular smooth muscle $\mathrm{IP}_{3}$ was shown to activate a plasma membrane cation channel which led to depolarization, opening of voltage-gated $\mathrm{Ca}^{2+}$ channels, and constriction. shRNA approaches indicated a role for $\mathrm{TRPC}_{3}$ both in isolated cells and pressurized arteries [21]. Leblanc similarly prevented evidence for a novel ion channel-based mechanism in pulmonary arterial smooth muscle cells [22]. Specifically, it was shown that $\mathrm{Ca}^{2+}$-activated $\mathrm{Cl}^{-}$currents could be activated in parallel with a store-operated non-selective cation current ( $\left.\mathrm{I}_{\mathrm{SOC}}\right)$.

Another dominant theme of the meeting related to sub-cellular $\mathrm{Ca}^{2+}$ handling and rhythmicity, or frequency-dependent events, in arterial smooth muscle. While not in resistance vessels, Imiataz and van Helden [23] presented a detailed model of pacemaking in lymphatic smooth muscle which will no doubt provide clues for the resistance artery field particularly with regard to arterial vasomotion. Lymphatic pacemaking was shown to utilize inositol-1,4,5triphosphate $\left(\mathrm{IP}_{3}\right)$ receptor-coupled $\mathrm{Ca}^{2+}$ stores synchronized as coupled oscillators [24]. 
Coupling of stores along the lymphatic vessel involved membrane depolarization and L-type $\mathrm{Ca}^{2+}$ channels. Interestingly, blockade of L-type $\mathrm{Ca}^{2+}$ channels with nifedipine, while blocking vasomotion, does not prevent asynchronous $\mathrm{Ca}^{2+}$ waves in either lymphatics [23] or arteriolar smooth muscle [25]. Further developing the vasomotion theme Terui (JVR citation) and Matchkov [26] presented novel evidence that vasomotion enhances tissue-capillary fluid exchange and that ICl,cGMP is mediated by bestrophin-4 and which may be important for vasomotion, respectively. Using elegant imaging and caged-compound approaches in single smooth muscle cells, McCarron [27] highlighted the complexity of the intracellular $\mathrm{Ca}^{2+}$ stores. A model was introduced where the SR exists as a luminously-continuous $\mathrm{Ca}^{2+}$ store rather than a series of discrete compartments. However, functional diversity arises from the distribution of $\mathrm{IP}_{3}$ and ryanodine receptors and their regulation by luminal $\left[\mathrm{Ca}^{2+}\right][28]$.

The contribution of oxidative stress to inflammation and vascular dysfunction was covered by a number of speakers throughout the ISRA meeting In the session dedicated to the subject Clark [29] stressed the importance of BOXes (Bilirubin Oxidation products) as important contributors to cerebral vasospasm in patients following hemorrhagic strokes. Kennedy [30] focused on the protease activated receptor-2 (PAR-2) and its contribution to neointima formation, which was much reduced in vascular injury studies with the PAR-1 knockout mouse. Zhang [31] stressed the importance of $\mathrm{NFKB}$ and TNF signaling in amplifying diabetes-associated endothelial dysfunction. Thomas [32] described the potentially important role that the leukocyte enzyme, myeloperoxidase (MPO) has, when associated with the endothelial cell, in inducing oxidative stress and endothelial dysfunction. Thus, blocking MPO sequestration into the endothelium is a potential target for protecting endothelial function. von der Weid extended the focus on inflammation to show that in a model of inflammatory bowel disease that lymphatic 
contractile activity was impaired [33]. The inhibition of lymphatic pumping was attenuated by cyclooxygenase inhibition suggesting a role for prostaglandins.

The role of the resistance vasculature in vascular disease was further considered in sessions relating to diabetes and hypertension. Jenkins [34], Triggle [34], Vanhoutte [35], Plane [36], focused on the effects of type 1 and type 2 diabetes on microvascular function with data presented from both human and animal studies. Jenkins emphasized the importance of the measurement of biomarkers as indicators of the impact of diabetes on cardiovascular function in humans as well as the efficacy of lifestyle and therapeutic interventions [34]. Complication-prone patients have comparatively higher levels of inflammation, oxidative stress, insulin resistance and tissue Advanced Glycation End-products (AGEs) burden as well as levels of the nitric oxide inhibitor, asymmetric dimethyl L-arginine (ADMA). Interestingly, comparable data is seen in animal models of type 1 diabetes thus supporting the argument that clinical and animal-based laboratory observations will facilitate the development of new approaches to the treatment as well as the prevention of diabetes-linked vascular disease. Triggle stressed the link between hyperglycaemia and, notably, the effects of acute changes in blood glucose (postprandial) on endothelial function with data indicating that elevated glucose resulted in a rapid "uncoupling" of eNOS that was possibly related to the oxidation of tetrahydrobiopterin [37]. Based on data from a cell culture assay with mouse microvascular endothelial cells exposure to elevated glucose also resulted in changes in expression of p22phox, eNOS, SOD1 and SOD3 indicating that the continued exposure of the endothelium to abnormal levels of glucose created a pro-oxidative stress environment that could be corrected by the provision of sepiapterin, a precursor of tetrahydrobiopterin. The effects of severe dyslipidaemia on the eNOS pathway, and cognitive function, were also stressed in a poster presentation by Drouin et al [38]. Triggle also noted that changes in the relative contributions of endothelium-derived nitric oxide versus the contribution 
and/or 'nature' of the endothelium-derived hyperpolarizing factor, EDHF, pathway also contributes to endothelial dysfunction [37]. Several presentations (Luksha et al., [39], Bansal et al., [40], Yang et al [41]) also provided evidence that alterations in the cellular processes contributing to EDHF are altered in cardiovascular disease. Vanhoutte focused on the link between vascular disease and endothelium-dependent increases in vascular tone that could be attributed to the release of Endothelium-Derived Contracting Factors - EDCFs [35]. The contribution of EDCFs to vascular disease has been noted in both animal and human studies and the resultant elevated vascular tone may result from a combination of the loss in the bioavailability of endothelium-derived nitric oxide as well as increases in the synthesis of EDCFs - angiotensin II, endothelin-1 as well as oxygen-derived free radicals and vascoconstrictor derivatives of arachidonic acid. Of importance was the argument that selective antagonists of prostanoid TP-receptors prevented endothelium-dependent contractions thus indicating the potential for such drugs in the treatment of endothelial dysfunction in not only diabetes but also hypertension. The poster presentation from Wong et al [42] also emphasized the contribution of COX-2-dependent EDCFs to acetylcholine-induced contractions in the hamster aorta. Plane presented data from streptozotocin (STZ)-induced diabetic rats that deficits in endotheliumdependent relaxation to acetylcholine in small mesenteric arteries and basilar arteries from the diabetic rat may be related to a dysfunction in the activation of endothelial intermediate conductance $\mathrm{K}_{\mathrm{Ca}}\left(\mathrm{IK}_{\mathrm{Ca}}\right)$ channels [36]. The provision of the activator of $\mathrm{IK}_{\mathrm{Ca}}$ channels, 1-ethyl-2benzimidazolinone (1-EBIO), restored acetylcholine-induced NO-dependent and -independent relaxations in vessels from diabetic rats, but quantitative RT-PCR showed no differences in the expression of either $\mathrm{IK}_{\mathrm{Ca}}$ or the small-conductance $\mathrm{SK}_{\mathrm{Ca}} 1-3$ channels. Thus, targeting the $\mathrm{IK}_{\mathrm{Ca}}$ may prove to be a novel target for treating diabetes-related vascular disease. Presentations from Haddock et al [43] and Brondrum et al [44] also supported the argument that changes in the 
regulation or distribution of $\mathrm{IK}_{\mathrm{Ca}}$ may be altered in vascular disease. Thorin [45] provided data from studies with both animal and human endothelial cells that linked endothelial cell senescence with cardiovascular disease and also linked smoking with a loss of antioxidant reserve and stressinduced senescence (SIS). Endothelial senescence was associated with a rise in markers of oxidative stress, such as HNE (4-hydroxy-2-nonenal), caveolin-1 expression as well as telomere shortening. These data indicated the importance of the redox environment and that an un-buffered bursts of free radical release results in accumulated damage. Of interest to was data from dyslipidemic mice that indicated that indicated that dyslipidemia hastened endothelial senescence and dysfunction and that chronic treatment with the antioxidant polyphenol catechin slowed progression of the indices of endothelial dysfunction.

Plasticity of the resistance vasculature was considered in the context of remodeling events which occur during physiological and pathophysiological conditions. Osol [46] described the physiological remodeling of the rat uterine circulation during pregnancy and then considered the added effects of NO inhibition (via L-NAME treatment) and increased perfusion pressure per se. While pregnancy caused marked effects on both axial remodeling (lengthening of the uterine artery and its branches) and circumferential remodeling of radial arteries, hypertension exerted a negative effect on only the circumferential changes. Van Bavel [47] described arteriolar organ culture studies underlying eutrophic remodeling responses to increased pressure and flow reduction. A sequence of events was proposed involving an initial inflammatory response and subsequent action of transglutaminase enzymes which modify the vessel wall by amino acid cross-linking reactions. Involvement of multiple members of this family of enzymes was suggested by data obtained in a transglutaminase 2 knockout model. Providing a link between mechanical forces and remodeling of the vessel wall, Pohl [48] presented studies showing that endothelial shear stress leads to elastase-mediated modification of the extracellular matrix, 
activation of $\mathrm{v}_{3}$ integrins and release of basic fibroblast growth factor.

A topic new to the ISRA program was the impact of the fetal environment on vascular (dys)function in later life. Considerable interest has developed over recent years in the concept of 'fetal programming' contributing to adult vascular disease including hypertension (for recent reviews see Poston [49] and Nuyt [50]). Davidge described animal model studies linking intrauterine hypoxia to endothelial dysfunction in adult offspring along with decreased cardiac tolerance to an ischemia/reperfusion event [51]. Leading on from this presentation several papers examined the effects of factors often found in the gestational environment, including alcohol [52], nicotine [53] and glucocorticoids [54], on vascular function of the offspring. Parkington reported that fetuses exposed to alcohol showed marked increases in arterial stiffness with extrapolations to large vessels thus suggesting that children with fetal alcohol syndrome would be more susceptible to atherosclerosis [52]. Similarly Tare concluded that prenatal glucocorticoid exposure resulted in endothelial dysfunction and pro-vasoconstrictor thus predisposing the offspring to lifelong cardiovascular dysfunction [54]. Lee described the detrimental effects of nicotine exposure in the fetus and neonate on perivascular adipose tissue (PVAT) function that resulted in a reduction in blood pressure lowering contribution of PVAT [53].

A vital part of the $9^{\text {th }}$ ISRA was sessions dedicated to Young Investigators. Outstanding oral presentations were made by Jonathan Ledoux, University of Vermont, Rebecca Haddock, University of New South Wales and Henrik Kold-Petersen, University of Aarhus. Ledoux (briefly mentioned above), demonstrated an association of calcium 'pulsar' release at internal elastic lamina hole sites, which are a prerequisite for myoendothelial gap junctions and correspond to sites of $\mathrm{IK}_{\mathrm{Ca}}$ and $\mathrm{IP}_{3} \mathrm{R}$ localization $[4,6,55]$ [7]. Such results support a novel mechanism of IP3-calcium signaling which is likely critical for vascular heterocellular signalling. 
Haddock highlighted changes in vasodilator function in disease, with a focus on diet-induced obesity [43]. Endothelium-dependent hyperpolarization (EDH) being due to activation of small and intermediate conductance calcium-activated potassium channels $\left(\mathrm{S} / \mathrm{IK}_{\mathrm{Ca}}\right)$ in normal rat mesenteric artery, and $\mathrm{IK}_{\mathrm{Ca}}$ alone in obese rat. Extending this theme, Kold-Petersen presented data examining the influence of $\mathrm{S} / \mathrm{IK}_{\mathrm{Ca}}$ activation with $\mathrm{NS} 309$ on the impaired EDH response of small arteries from the Zucker diabetic fatty rat (model of Type 2 diabetes) [44]. The $\mathrm{K}^{+}$channel activator was found to improve EDH-dependent dilation independently of an effect on intracellular $\mathrm{Ca}^{2+}$. Additional studies performed with apamin and TRAM-34 suggested that the improvement occurred largely at the level of $\mathrm{IK}_{\mathrm{Ca}}$.

While the meeting began under monsoon conditions the science shone through and eventually perfect weather followed. After successful completion of the $9^{\text {th }}$ ISRA plans have begun for the $10^{\text {th }}$ ISRA to be held in Denmark in 2011. As a sad footnote to the meeting the ISRA community would like to acknowledge the immense contributions of Dr William Halpern (1923 - 2008). He was an 'ISRA Founder' and a vital contributor both through his science and support through generous sponsorship provided by his company. Bill will be missed. 


\section{References}

1 Beny J-L: Conduction of signals along the vascular wall. ISRA Communication 2008

2 de Wit C: Communication along the vascular wall: Distinct connexins and pathways involved. Journal of Vascular Research 2008;45:2.

3 Wolfle SE, Schmidt VJ, Hoepfl B, Gebert A, Alcolea S, Gros D, de Wit C: Connexin45 cannot replace the function of connexin40 in conducting endothelium-dependent dilations along arterioles. Circ Res 2007;101:1292-1299.

4 Ledoux J, Taylor MS, Bonev AD, Hannah RM, Tallini YT, Kotlikoff MI, Nelson MT: $\mathrm{Ca} 2+$ pulsars: Spatially restricted, ip3-mediated ca2+ release important for endothelial function. Journal of Vascular Research 2008;45:3.

5 Tallini YN, Brekke JF, Shui B, Doran R, Hwang SM, Nakai J, Salama G, Segal SS, Kotlikoff MI: Propagated endothelial ca2+ waves and arteriolar dilation in vivo: Measurements in cx40bac gcamp2 transgenic mice. Circ Res 2007;101:1300-1309.

6 Ledoux J, Taylor MS, Bonev AD, Hannah RM, Solodushko V, Shui B, Tallini Y, Kotlikoff MI, Nelson MT: Functional architecture of inositol 1,4,5-trisphosphate signaling in restricted spaces of myoendothelial projections. Proceedings of the National Academy of Sciences of the United States of America 2008;105:9627-9632.

$7 \quad$ Sandow SL: Clinical and Experiental Physiology and Pharmacology 2008; in press

8 Isakson BE: Imaging calcium dynamics at the myoendothelial junction in vitro. Journal of Vascular Research 2008;45:9.

9 Brayden J: Transient receptor potential (trp) channels, vascular tone and blood flow regulation. Journal of Vascular Research 2008;45:13.

10 Hill MA, Sun Z, Ella SR, Meininger GA: New imaging approaches for understanding the arteriolar myogenic response. Journal of Vascular Research 2008;45:23.

11 Reading SA, Brayden JE: Central role of trpm4 channels in cerebral blood flow regulation. Stroke; a journal of cerebral circulation 2007;38:2322-2328.

12 Scholfield CN, Curtis TM, McGeown JG: Cell ca2+ and ca2+-activated channels in retinal arterioles. Journal of Vascular Research 2008;45:58.

13 Jackson WF: Regulation of bkca channels in arteriolar smooth muscle cells. Communication 2008 
14 Hwang YT, Haddock RE, Hill MA, Murphy TV: Heterogenous role of bkca in modulating myogenic tone in arterioles? Journal of Vascular Research 2008;45:30.

15 Martinez-Lemus LA, Wu X, Wilson E, Hill MA, Davis GE, Davis MJ, Meininger GA: Integrins as unique receptors for vascular control. J Vasc Res 2003;40:211-233.

16 Sun Z, Martinez-Lemus LA, Hill MA, Meininger GA: Extracellular matrix-specific focal adhesions in vascular smooth muscle produce mechanically active adhesion sites. Am J Physiol Cell Physiol 2008;295:C268-278.

17 Bolz SS: The role of sphingosine-1-phosphate (s1p) signalling for the regulation of resistance artery tone under physiological and pathophysiological conditions. Journal of Vascular Research 2008;45:22.

18 Peter BF, Lidington D, Harada A, Bolz HJ, Vogel L, Heximer S, Spiegel S, Pohl U, Bolz SS: Role of sphingosine-1-phosphate phosphohydrolase 1 in the regulation of resistance artery tone. Circ Res 2008;103:315-324.

19 Matchkov VV, Larsen P, Kold-Petersen H, Bouzinova EV, Boedtkjer D, Andresen J, Aalkjaer C: Sirna-induced in vivo down regulation of 1-type calcium channels in rat small mesenteric arteries. Journal of Vascular Research 2008;45:15.

20 Jaggar JH: Ip3 receptors modulate vascular contractility through cation channel activation. Journal of Vascular Research 2008;45:13a.

21 Xi Q, Adebiyi A, Zhao G, Chapman KE, Waters CM, Hassid A, Jaggar JH: Ip3 constricts cerebral arteries via ip3 receptor-mediated trpc3 channel activation and independently of sarcoplasmic reticulum ca2+ release. Circ Res 2008;102:1118-1126.

22 Leblanc N: Functional significance of a new coupling mechanism between ca2+-activated cl- channels and store-operated ca2+ entry in mammalian pulmonary arterial smooth muscle cells. Journal of Vascular Research 2008;45:14.

23 Imtiaz MS, vanHelden DF: Pacemaking in lymphatic smooth muscle. Journal of Vascular Research 2008;45:59.

24 Imtiaz MS, Zhao J, Hosaka K, von der Weid PY, Crowe M, van Helden DF: Pacemaking through ca2+ stores interacting as coupled oscillators via membrane depolarization. Biophysical journal 2007;92:3843-3861.

25 Ella SR, Davis MJ, Dora KA, Meininger GA, Hill MA: Interactions between intraluminal pressure, myogenic tone and smooth muscle ca2+ oscillations in cremaster muscle 
arterioles. Journal of Vascular Research 2008;45:28.

26 Matchkov VV, Larsen P, Bouzinova EV, Boedtkjer D, Andresen J, Nilsson H, Aalkjaer

C: Importance of bestrophin-4 protein for vasomotion. Journal of Vascular Research 2008;45:61.

27 McCarron JG: A single luminally-continuous sarcoplasmic reticulum with apparently separate ca2+ stores in smooth muscle. Journal of Vascular Research 2008;45:37.

28 McCarron JG, Olson ML: A single luminally continuous sarcoplasmic reticulum with apparently separate ca2+ stores in smooth muscle. The Journal of biological chemistry 2008;283:7206-7218.

29 Wurster WL, Loftspring M, Pyne-Geithman GJ, Clark JF: Bilirubin oxidation products (boxes) are produced by biochemical oxidation and oxidative stress and may contribute to vascular dysfunction. Journal of Vascular Research 2008;45:69.

30 Kennedy S: Is par-2 involved in formation of neointima? Journal of Vascular Research 2008;45:70.

31 Yang J, Park Y, Dellsperger KC, Zhang C: Feed-forward signaling of tnf and nfkb produces endothelial dysfunction in coronary arterioles in type 2 diabetic mice. Journal of Vascular Research 2008;45:71.

32 Thomas SR: Oxidative stress and endothelial dysfunction. Journal of Vascular Research 2008;45:72.

33 von der Weid P-Y: Impairment of lymphatic contractile function during intestinal inflammation. ISRA Communication 2008

34 Jenkins AJ: Markers and mediators of the vascular complications of diabetes mellitus. Journal of Vascular Research 2008;45:89.

35 Vanhoutte PM: Endothelial dysfunction and vascular disease: Edcf revisited. Journal of Vascular Research 2008;45:91.

36 Plane F: Activation of endothelial ca2+-activated potassium channels can improve endothelial function in arteries from diabetic rats. Journal of Vascular Research 2008;45:93.

37 Triggle CR, Aljofan M, Cheng Z-J, Pannirselvam M, Li Y, Hollenberg M, Ding H: Endothelial dysfunction and diabetic microvascular disease: The demise of no alone or does edhf alos change? Journal of Vascular Research 2008;45:90. 
38 Drouin A, Belanger P, Ferland G, Thorin E: Chronic antioxidant therapy prevents mouse cerebral endothelial dysfunction associated with dyslipidemia and improves cognitive function. Journal of Vascular Research 2008;45:97.

39 Luksha L, Lang NN, Nisell H, Newby DE, Kublickiene K: Altered edhf-type responses in small arteries from women with preeclampsia. Journal of Vascular Research 2008;45:101.

40 Bansal M, Lau W, Young EJ, Reid JJ: Edhf-mediated relaxations in renal arteries from type-1 diabetic rats: Relative contribution of calcium-activated potassium channels. Journal of Vascular Research 2008;45:103.

41 Yang Q, Shigemura N, Hsin M, Wong W-T, Tian X-Y, Huang Y, Yim APC, He G-W: Pulmonary endothelial function in copd (chronic obstructive pulmonary disease) patients of different severity: Studies on both arteries and veins. Journal of Vascular Research 2008;45:105.

42 Wong W-T, vanhoutte PM: ISRA Communication 2008

43 Haddock RE, Howitt L, Morris MJ, Sandow SL: Changes in kca subtype activity may underlie altered endothelium-derived hyperpolarization in diet-induced obese rat mesenteric artery. Journal of Vascular Research 2008;45:63.

44 Brondrum E, Kold-Petersen H, Aalkjaer C: Ns309 restores edhf mediated relaxation in isolated mesenteric small arteries from the zdf rat model of type 2 diabetes. Journal of Vascular Research 2008;45:64.

45 Thorin-Trecases N, Gendron M-E, Voghel G, Farhat N, Nguyen A, Thorin E: Risk factors, oxidative stress, endothelial cell dysfunction and senescence. Journal of Vascular Research 2008;45:94.

46 Osol G: Remodeling of the uterine circulation during pregnancy: Differential effects of hypertension vs nitric oxide inhibition. Journal of Vascular Research 2008;45:81.

47 van Bavel E: Small artery inward remodeling: Role of transglutaminases and inflammation. Journal of Vascular Research 2008;45:83.

48 Pohl U: Shear stress dependent fgf-2 release from endothelial cells is mediated by elastase activity and integrin dependent signaling pathways. Journal of Vascular Research 2008;45:26.

49 Poston L: Influences of maternal nutritional status on vascular function in the offspring. Curr Drug Targets 2007;8:914-922. 
50 Nuyt AM: Mechanisms underlying developmental programming of elevated blood pressure and vascular dysfunction: Evidence from human studies and experimental animal models. . Clin Sci 2008;114:1-17.

51 Davidge S: Long term cardiovascular consequences for offspring born in an adverse in utero environment. Journal of Vascular Research 2008;45:107.

52 Parkington HC, Coleman HA, Kenna K, Walker D, Harding R, Tare M: Maternal alcohol in pregnancy induces a marked, generalized increase in arterial stiffness and alters endothelial vasodilator function in the fetus. Journal of Vascular Research 2008;45:108.

53 Gao TJ, Holloway AC, Su LY, Takemori K, Lu C, Lee RMKW: Fetal and neonatal exposure to nicotine elevates blood pressure and alters perivascular adipose tissue function. Journal of Vascular Research 2008;45:110.

54 Tare M, Moritz KM, Jefferies AJ, Dodic M, Coleman HA, Parkington HC: Two days of glucocorticoid exposure early in gestation results in lifelong vascular dysfunction. Journal of Vascular Research 2008;45:109.

55 Sandow SL, Neylon CB, Chen MX, Garland CJ: Spatial separation of endothelial smalland intermediate-conductance calcium-activated potassium channels (k(ca)) and connexins: Possible relationship to vasodilator function? Journal of Anatomy 2006;209:689-698. 